\title{
Investigación-acción, aula invertida y formación basada en competencias. Elementos clave para una efectiva formación b-learning en liderazgo directivo
}

\section{Investigation-action, inverted classroom and training based on skills. Key elements for effective b-learning training in management leadership}

\author{
Rosita Romero Alonso \\ Investigadora, Centro de Innovación en Educación \\ Universidad Tecnológica de Chile INACAP \\ Santiago, Chile \\ reromero@inacap.cl \\ Blanca Montt Fabres \\ Investigadora, Centro de Innovación en Educación \\ Universidad Tecnológica de Chile INACAP \\ Santiago, Chile \\ blanca.montt@inacap.c \\ Claudio Maregatti Solano \\ Investigador, Centro de Innovación en Educación \\ Universidad Tecnológica de Chile INACAP \\ Santiago, Chile \\ cmaregatti@inacap.cl \\ María Elena Gracia Romero \\ Investigadora, Centro de Innovación en Educación \\ Universidad Tecnológica de Chile INACAP \\ Santiago, Chile \\ maria.gracia02@inacap.cl \\ Daniel Segura Ángel \\ Investigador, Centro de Innovación en Educación \\ Universidad Tecnológica de Chile INACAP \\ Santiago, Chile \\ dsegura@inacap.cl
}

Recibido - Received: 10 / 10 / 2019

Corregido - Revised: 10 / 02 / 2020

Aceptado - Accepted: 21 / 02 / 2020

DOI: https://doi.org/10.22458/ie.v22i32.2896

URL: https://revistas.uned.ac.cr/index.php/innovaciones

Resumen: Frente al desafío de contar con enfoques formativos para docentes y directivos en ejercicio que atiendan las complejidades del fenómeno educativo, es necesario evidenciar la efectividad de diversas metodologías formativas. El artículo describe los resultados de una investigación cuyo objetivo fue determinar la efectividad de un curso en liderazgo para la innovación y cambio en las instituciones escolares, orientado a docentes y directivos escolares en ejercicio, y conocer la valoración de éstos respecto a la estrategia didáctica aplicada y su articulación con las TIC, al identificar factores del diseño formativo que favorecen la transferencia de los aprendizajes al contexto educativo. Para ello, se utilizó una metodología cuantitativa, a través de la aplicación de un pre y posttest de conocimientos a una muestra de 168 participantes del curso; y luego una encuesta de satisfacción aplicada en tres cohortes, respondida por 123 participantes. Los resultados muestran una mejora estadísticamente significativa en los conocimientos sobre habilidades directivas de los participantes, lo cual evidenció la efectividad del curso. Por otra parte, el enfoque aplicado que orientó el curso, sustentado en la investigación-acción y el aula invertida desde una modalidad b-learning, fue altamente valorado por los docentes por cuanto propicia actividades aplicadas y contextualizadas, trabajo autónomo conjugado con reflexión colaborativa. Lo que permite fortalecer la autoconfianza profesional, favoreciendo a la vez la posterior transferencia de los aprendizajes.

Palabras clave: Educación continua; formación de profesores en activo; aprendizaje semipresencial; liderazgo directivo 
Summary: Faced with the challenge of having training approaches for practicing teachers and managers that address the complexities of the educational phenomenon, it is necessary to demonstrate the effectiveness of various training methodologies. The article describes the results of an investigation the objective of which was to determine the effectiveness of a course in leadership for innovation and change in school institutions, aimed at practicing teachers and school managers. The investigation also wanted to learn their assessment regarding the applied teaching strategy and its articulation with ICTs, by identifying factors of the training design that favor the transfer of learning to the educational context. For this, a quantitative methodology was used, through the application of a pre and post-test of knowledge to a sample of 168 course participants; and then a satisfaction survey applied in three cohorts, answered by 123 participants. The results show a statistically significant improvement in the participants' knowledge of management skills, which evidenced the effectiveness of the course. On the other hand, the applied approach that guided the course, based on action research and the inverted classroom from a b-learning modality, was highly valued by teachers as it encourages applied and contextualized activities, autonomous work combined with collaborative reflection. This makes it possible to strengthen professional self-confidence, while favoring the subsequent transfer of learning.

Keywords: Continuing education; active teacher training; blended learning; managerial leadership

\section{INTRODUCCIÓN}

En la actualidad, la formación docente se concibe como un proceso continuo y es considerada un factor relevante en la calidad de los aprendizajes de los alumnos (Hattie, 2009; Nieto \& Alfageme-González, 2017). Transformarla puede impactar profundamente los sistemas educativos. Bajo este contexto, las instituciones formadoras se ven desafiadas a diseñar procesos de enseñanza y aprendizaje que integren lo que los estudios señalan respecto a las mejores modalidades y enfoques (Nieto \& Alfageme-González, 2017), dejando atrás modelos de formación que acarrean una insatisfacción docente frente a los procesos formativos (Díaz Larenas, Solar Rodríguez, \& Soto Hernández, 2015).

En Chile, se observa como, por un lado, se han hecho avances ostensibles bajo la Ley №20.903 (2016) que creó el Sistema de Desarrollo Profesional Docente para asegurar el acceso a formación, mientras que, por otro, la calidad de los programas es cuestionada por el profesorado. Así, en el análisis chileno del informe TALIS 2013 (Barrera, Castillo, \& Gallardo, 2017), se reporta que profesionales de la educación indican como la segunda barrera para un mayor desarrollo profesional el que no se ofrezcan oportunidades adecuadas de formación.

En este contexto, surge la necesidad de observar modelos formativos que atiendan las inquietudes del profesorado y cumplan efectivamente con la función de desarrollar nuevas competencias. El sistema demanda metodologías de formación pertinentes y con validez de contenido para quienes participan en las actividades formativas (Grohmann, Beller, \& Kauffeld, 2014; Grohmann \& Kauffeld, 2013), las cuales faciliten la posterior puesta en práctica de las nuevas competencias en el contexto educativo donde se desempeñan. Por tanto, se propone como objetivo en la investigación determinar la efectividad de un curso blended-learning (semipresencial), en liderazgo para la innovación y cambio en las instituciones escolares, y conocer la valoración de los egresados respecto a la metodología en la que se sustenta el curso, identificando los factores del diseño formativo utilizado en esta modalidad $b$-learning que favorecen la transferencia de los aprendizajes al contexto educativo.

\section{MARCO TEÓRICO}

\section{Desarrollo de competencias, contexto y transferencia de aprendizajes}

Un aspecto interesante de los diseños formativos basados en competencias es que valorizan fuertemente la contextualización de las acciones de aprendizaje. Considera al contexto como un elemento crucial pues en él es donde se ponen en juego conocimientos, habilidades y actitudes para el logro de la tarea, 
permitiendo a su vez reconocer nuevos espacios de aplicabilidad de las mismas (Cano, 2008; Perrenaud, 2004; Tobón, 2013). Bajo esta perspectiva, la formación no puede estar disociada de la acción (Le Boterf, 2011). Se deben articular estrategias orientadas a la acción para visibilizar y anticipar el desarrollo de las competencias (Tejada Fernández, 2012).

Para este enfoque, la organización de las tareas en torno a acciones que permitan observar desempeños es relevante (Romero \& Halal, 2017; Tobón, 2013). El centro es el estudiante, los desempeños de éste, las acciones desarrolladas por él en un contexto de pertinencia, actualizado y de autenticidad (Tejada Fernández \& Ruiz Bueno, 2016).

A su vez, desde la mirada de la formación en ejercicio de la profesión, la contextualización de los procesos formativos es relevante para las posibilidades de transferir el conocimiento aprendido a las prácticas profesionales, especialmente gracias a la autoconfianza que adquieren los aprendices (Grohmann, Beller, \& Kauffeld, 2014; Grohmann \& Kauffeld, 2013; Hutchins, Burke, \& Berthelsen, 2010). Bajo este prisma, los diseños formativos basados en aprendizaje activo han demostrado efectividad (Gegenfurtner, 2011).

Si pensamos en el diseño de una formación en liderazgo para transferir, se debe considerar un acompañamiento docente que permita un seguimiento y un clima ideal para trabajar colaborativamente, socializando los aciertos y los obstáculos (Cano, 2016; Lepe, Castillo, Cerda, Montaña, \& Recabarren, 2014). Aspectos que fueron nuevamente destacados en un estudio desarrollado en Chile sobre la formación de directivos escolares, donde los propios exalumnos subrayan la importancia de la pertinencia de los métodos formativos a la realidad escolar y las posibilidades de construir redes que permitan luego el mantenimiento de los aprendizajes (Azocar \& Cano, 2015). Por lo tanto, la transferencia de aprendizajes al contexto requiere que los diseños formativos sean flexibles y permitan a los formadores vincularse con la realidad de los alumnos y a los agentes participantes motivarse en un compromiso al cambio, basado en la valorización de su entorno (Cano, 2015).

Las formaciones más efectivas son aquellas desarrolladas in situ y basadas en un conocimiento que valora el aporte de las comunidades de práctica (Amador \& Pagés, 2014; Feixas, Marquez, \& Sabaté, 2014). Generar una cultura de transferencia y de reflexión sobre la práctica es un nuevo desafío por integrar en los procesos formativos (Cano, 2015), y claramente en la formación de directivos escolares.

\section{La formación a través de programas b-learning y el enfoque de aula invertida}

El blended learning, conocido como aprendizaje híbrido en sus comienzos y hoy identificado como aprendizaje semipresencial, corresponde a una modalidad de enseñanza que mezcla actividades de aprendizaje guiadas en clases presenciales y otras desarrolladas en ambientes virtuales de aprendizaje (Bartolomé, 2004; Garrison, 2011).

En un proceso blended learning se espera sumar las ventajas del e-learning, tales como mayor accesibilidad, la flexibilidad y la eficiencia de los costos, al sentido de pertenencia a una comunidad y al acompañamiento que son propios de la modalidad presencial (Bocconi \& Trentin, 2014). Algunos estudios señalan que implementar modalidad blended learning ha mejorado el aprendizaje (Faturrahman, Ibrahim, Kahar, Arsyad, \& Rawi, 2018) y la motivación (Cui \& Hu, 2018) de los participantes.

Optar por el aprendizaje en formato blended learning no implica simplemente la integración del componente tecnológico o virtual, sino que debe también transformar las estrategias, la utilización de los recursos de infraestructura e incluso reducir la mera transmisión de conocimientos en lo presencial (Simon, Benedí, Blanche, \& Bosch, 2016). 
Otros aspectos por cuidar son la mediación, las características del equipo formador y la integración de estrategias de aprendizaje aplicadas (Dewi, Ciptayani, Surjono, \& Priyanto, 2018; Goode et al., 2018; Martin, Kumar, Abernathy, \& Browne, 2018; Yeop, Yaakob, Wong, Don, \& Zain, 2019).

Últimamente, en el ámbito de los programas blended learning se ha posicionado una modalidad identificada como flipped classrrom o aula invertida, que consiste en integrar tecnologías, especialmente audiovisuales, combinadas con la formación presencial dedicada al desarrollo de actividades de aplicación (Bergmann \& Sams, 2012; Limniou, Schermbrucker, \& Lyons, 2018). Se considera que este enfoque permite una mayor interacción entre formador y aprendices, así como con el propio material de aprendizaje, tanto dentro como fuera del tiempo de clases presenciales (Gilboy, Heinerichs, \& Pazzaglia, 2015).

No es en la clase, sino en los espacios de interacción virtual donde se puede profundizar conceptualmente (Strayer, 2012). Esta característica permite a quienes guían las actividades dedicar tiempo al desarrollo de actividades de aprendizaje activas (Bergmann \& Sams, 2012), y a los estudiantes comprometerse siguiendo un proceso de aprendizaje flexible con opciones de acceder a recursos más libremente y a un enfoque creativo e innovador (Herreid \& Schiller, 2013). Además, el tipo de estrategias utilizadas, tal como la discusión grupal, permite a los alumnos dedicar tiempo al aprendizaje de orden superior (Zainuddin \& Halili, 2016).

Claramente, el éxito del enfoque del aula invertida depende en gran medida del diseño de las clases (Lee \& Lai, 2017). El rol de la elección del material de aprendizaje, las actividades y las características de los maestros es preponderante (Limniou et al., 2018).

\section{La investigación-acción como vehículo para el cambio de prácticas}

La investigación-acción (IA) concebida como un proceso investigativo que se orienta a la mejora de las prácticas educativas y que busca decididamente el cambio de éstas (Carr, 1993; Rowell, Polush, Riel, \& Bruewer, 2015), es también una perspectiva que puede ser considerada de formación en la medida que permite desarrollar aprendizajes sobre la propia práctica educativa (Kemmis \& McTaggart, 1988). La IA y los procesos reflexivos que desarrolla, generan conocimiento metacognitivo y practican fuertemente la solución de problemas, ambas habilidades consideradas formas de pensamiento de orden superior (Van Velzen, 2017).

Aspectos destacados propios de un proceso de IA identifican cambios en las prácticas o en las creencias, la posibilidad de reflexión sobre sus problemas, la búsqueda de soluciones innovadoras, el trabajo colaborativo, la toma de decisiones informada, el desarrollo de la capacidad de crítica y la posibilidad de aprender de la propia práctica (Díaz-Bazo, 2017). Una investigación reciente sobre procesos de IA en conservatorios de música (Campayo-Muñoz \& Cabedo-Mas, 2018), refuerza el valor de seminarios impartidos sobre IA por pares como un recurso que promueve la reflexión y transformación de la práctica docente.

Se ha mostrado el valor de la IA para la formación de formadores de lenguas extranjeras en educación superior potenciando su rol investigador, cuya experiencia destaca la posibilidad de trabajo en equipo y con la guía de un tutor que los oriente como fortalezas para un proceso formativo de este tipo (Burns \& Westmacott, 2018).

Más recientemente, los hallazgos de un programa para formar a directores de escuela noruegos, basado en el método de investigación-acción, ha mostrado cómo un programa formativo de este tipo puede ser concebido como un proceso sistemático y disciplinado, que apoya cambios en las prácticas, en la comprensión de los fenómenos y creencias sobre liderazgo directivo (Aas, Vennebo, \& Halvorsen, 2019). En particular, el estudio atribuye al involucramiento práctico y prueba de nuevas prácticas de liderazgo por los directivos el factor clave que provocaría las transformaciones observadas. 


\section{METODOLOGÍA}

\section{Contexto de aplicación}

El estudio se enmarca en un curso Liderazgo para la Innovación y Cambio en las Instituciones Escolares que forma parte de la oferta de actualización para profesionales de la educación del Centro de Perfeccionamiento, Experimentación e Investigaciones Pedagógicas (CPEIP) del Ministerio de Educación de Chile, la cual busca desarrollar conocimientos, competencias y prácticas directivas para mejorar la gestión escolar y el aprendizaje de los estudiantes. La ejecución de los cursos está a cargo de instituciones de educación superior siguiendo orientaciones generales para su ejecución. El estudio se centra en un curso con modalidad b-learning, que tiene 251 horas de duración distribuidas en seis meses, donde participaron docentes (aspirantes a cargos directivos) y directivos, que llevan entre uno y tres años en el cargo, todos de la zona norte del país.

En concreto, el curso se propone desarrollar competencias para conducir y liderar procesos de mejora educativa, a partir del conocimiento sobre política educativa, innovación, desafíos del liderazgo escolar y de los principios pedagógicos que resguardan la calidad de la enseñanza. El itinerario formativo se desarrolla en el ámbito virtual, donde quienes participan del curso adquieren y profundizan los conocimientos a través de diversas actividades tanto individuales como grupales, guiados por un experto. En el ámbito presencial, a través de talleres y grupos de discusión guiados por un tutor, lo cual potencia el trabajo colaborativo de los participantes, ofrece oportunidades para compartir experiencias, hacer análisis críticos de sus contextos y realizar procesos metacognitivos de su aprendizaje. Así como en sus propios contextos educativos por medio de la aplicación de una investigación-acción (IA) que les permite vivenciar, en su centro, estrategias para conducir y liderar procesos de mejora educativa.

Figura 1. Metodología de formación b-learning curso de Liderazgo Directivo.

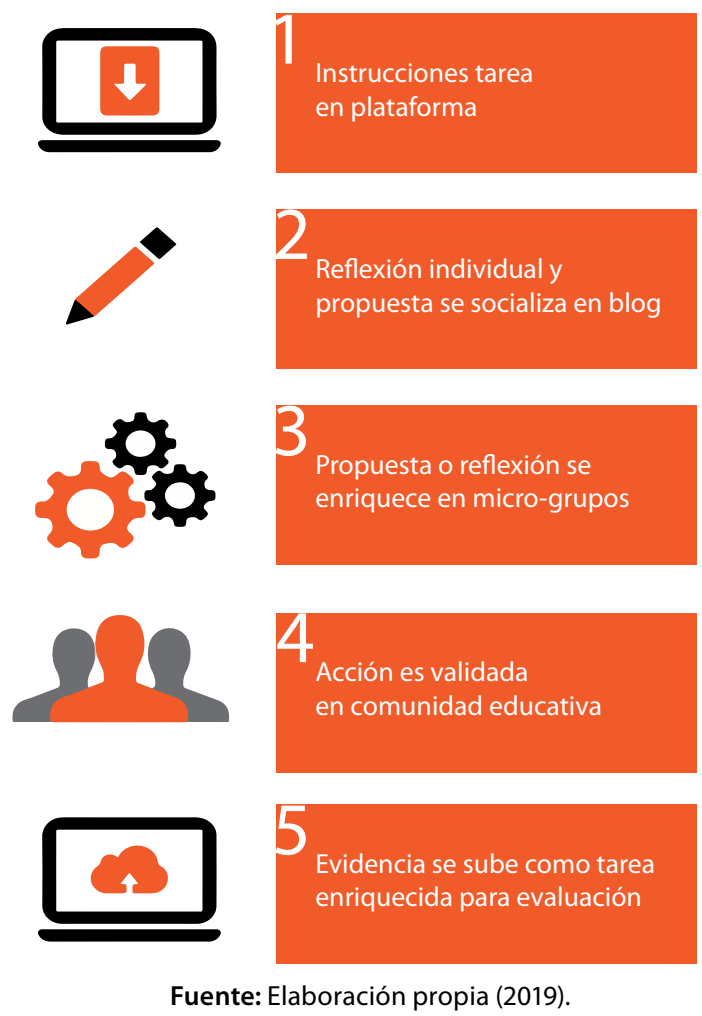




\section{Diseño}

Se trata de una investigación con un enfoque cuantitativo, basado en un diseño transversal con una metodología descriptiva y correlacional. En función de los objetivos se construyeron dos instrumentos para recoger información. El primero de ellos, una prueba de conocimientos que se aplicó al iniciar el curso (pre-test) y al finalizarlo (post-test) a todos los participantes, y permitió determinar la efectividad del curso en función de los aprendizajes evidenciados. La prueba tiene un total de 32 reactivos distribuidos en las temáticas abordadas en cada módulo del curso, donde $34,4 \%$ de los reactivos se clasifican en el nivel cognitivo de recordar, $37,5 \%$ en comprender y $28,1 \%$ en aplicar.

Se aplicó el mismo instrumento de evaluación de conocimientos en tres versiones del curso (2016/ 2017/2018). Para el análisis, se consideraron solo a quienes participaron en ambas evaluaciones, logrando una muestra representativa de 168 personas, con un margen de error de 1,5\% con un nivel de confianza de $95 \%$.

En segundo término, se diseñó un cuestionario estructurado autoadministrado, vía plataforma online (Díaz de Rada \& Domínguez Álvarez, 2017), orientado a conocer la satisfacción de quienes participaron del curso en cuanto las metas y objetivos del curso, los docentes, los contenidos, la metodología, las actividades y las tareas, el sistema de evaluación, los recursos y los materiales, el proyecto aplicado y las sesiones presenciales con los tutores.

El instrumento consta de 68 ítemes en escala Likert de 1 a 5 (muy de acuerdo; de acuerdo; ni de acuerdo ni en desacuerdo; en desacuerdo; totalmente en desacuerdo), además de cuatro preguntas de caracterización sociodemográfica del docente, y tres preguntas abiertas de valoración general del curso. El cuestionario tiene una alta consistencia interna en la prueba de Alpha de Cronbach $(0,95)$. Su aplicación se efectuó al finalizar el curso, en su versión 2016, 2017 y 2018.

En sus tres momentos de aplicación, se logró una alta tasa de respuesta pues se consiguió una muestra total de 123 sujetos, con un margen de error de 4,8\% con un nivel de confianza de $95 \%$. Así, se obtiene una muestra representativa donde todos los encuestados tienen formación en educación y ejercen en establecimientos escolares que reciben aportes públicos desde el Estado (municipales o particulares subvencionados), y se desempeñan en escuelas primarias y secundarias. La mayoría (56,6\%) tiene más de 15 años de experiencia docente, por lo que se está frente a profesionales de la educación caracterizados por la estabilidad profesional de acuerdo con la clasificación de Michael Huberman (1990). Al momento de matricularse $43,3 \%$ ejerce un rol directivo en el centro educativo, contando en promedio con 2,5 años en el cargo. En cuanto a la distribución por género se observa una mayor cantidad de mujeres $(61,8 \%)$ que de hombres $(38,2 \%)$.

\section{RESULTADOS}

\section{Efectividad del curso}

En relación con la efectividad del curso, medida a través de un pre y post-test de conocimientos sobre liderazgo directivo, se aprecia diferencia entre las calificaciones medias en los tres años de ejecución del curso, siendo mayor en el post-test con $80,9 \%$ de logro, mientras que en el pre-test se observó $68,9 \%$ de logro. 
Figura 2. Comparación de resultados de aprendizajes

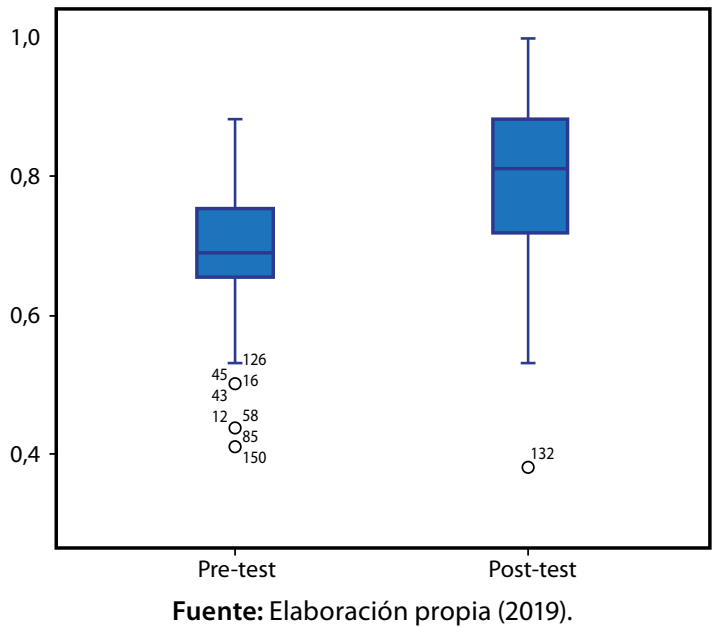

Para analizar si se trata de una diferencia estadísticamente significativa, se realizó comparación de medias. En primer lugar, las pruebas de normalidad (Test K-S y Test Shapiro Wilk) indicaron que los datos no presentan una distribución normal, por lo que se trabajó con la prueba $\mathrm{W}$ de Wilcoxon, la versión no paramétrica a la habitual prueba $\mathrm{T}$ de Student para muestras dependientes. Con hipótesis nula $\left(\mathrm{H}_{0}\right)$ : los resultados son iguales, y con la hipótesis alternativa $\left(\mathrm{H}_{1}\right)$ : los resultados son diferentes. Basados en el resultado de significación asintótica (bilateral) menor al valor alfa 0,01 , se rechaza $\mathrm{H}_{0}$. Es decir, se puede afirmar con 99\% de confianza que los resultados de las calificaciones difieren, siendo más altos los resultados obtenidos en el post-test.

De esta manera, como primer hallazgo se puede señalar que los profesores y directivos que participaron en el curso Liderazgo para la Innovación y Cambio en las Instituciones Escolares evidencian una mejora en sus conocimientos sobre liderazgo directivo una vez terminada la acción formativa. Ahora bien, para conocer de qué manera la metodología utilizada y los recursos tecnológicos dispuestos facilitaron el aprendizaje, se revisaron los principales resultados de la encuesta de satisfacción.

\section{Valoración del enfoque aplicado desde la perspectiva de los participantes}

Se observa una positiva valoración de la metodología en que se sustenta el curso, destaca que $88,6 \%$ de quienes participaron del curso está de acuerdo o muy de acuerdo con la articulación de los elementos que le dan vida: lo virtual y presencial, el desarrollo de un proyecto aplicado y la visita a la institución por parte del tutor.

Desde de la percepción cualitativa, desprendida desde las preguntas abiertas del cuestionario, se subrayan los elementos clave del curso, como su metodología y posibilidad de transferencia, así como el énfasis en la participación y reflexión "(...) es un curso de alto nivel y que mezcla equilibradamente la teoría y la práctica. Sus contenidos y metodologías son significativos y transferibles a los establecimientos" (Participante 55, Curso 2016).

Por su parte, el enfoque aplicado fue altamente valorado en tanto oportunidad de análisis del propio contexto institucional a través de la investigación-acción, 98\% de los encuestados valoran positivamente esta forma de trabajo como una oportunidad para desarrollar las habilidades directivas. 
Figura 3: Valoración del enfoque aplicado.

El enfoque aplicado, bajo la investigación-acción, ayudó al desarrollo de mis competencias directivas

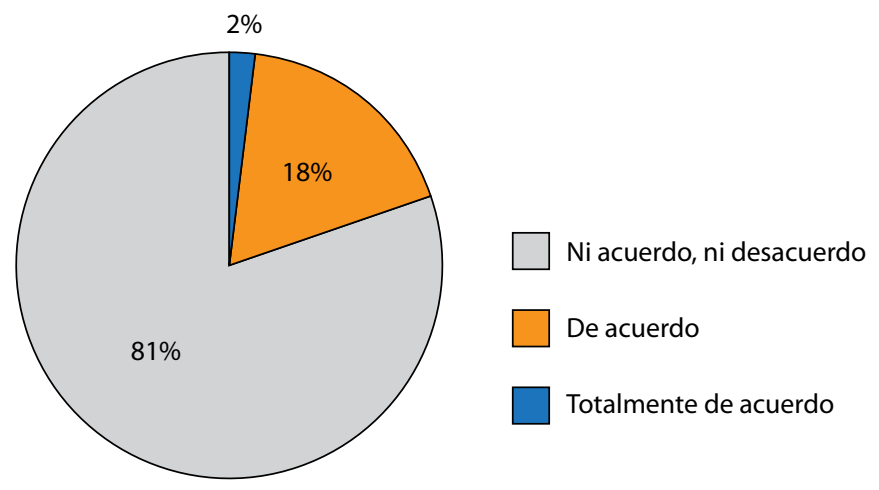

Fuente: Elaboración propia (2019).

En relación con el desarrollo de las competencias directivas, a partir de un enfoque aplicado se destaca que "el trabajo en equipo, logramos formar grupos de trabajo muy afiatados nos apoyamos y retroalimentamos constantemente. Lograr desarrollar competencias para mejorar nuestra gestión como directivos" (Participante 53, Curso 2016).

En este contexto, la reflexión sobre las prácticas pedagógicas/directivas y el entorno educativo se transforma en un eje articulador, la que se concretó a través de diversas tareas y actividades durante el curso, siendo altamente valorada. Así, 99,2\% considera que la reflexión sobre las tareas aportó a la comprensión del propio contexto institucional y 95,9\% que las actividades facilitaron la reflexión sobre la práctica y el contexto educativo; por tanto $97,6 \%$ considera que el curso le ha permitido reflexionar sobre sus prácticas pedagógicas/directivas. Por otra parte, es claro para los participantes que el curso implementó un enfoque orientado a la práctica, así 95,9\% señala que tuvo la posibilidad de aplicar los contenidos abordados en su propia práctica y contexto educativo, y 91\% pudo transferir la teoría a situaciones concretas del ámbito escolar. Especialmente, es observable cuando $99 \%$ consideró que la investigación-acción desarrollada en el contexto del curso contribuyó al análisis de un tema relevante del propio contexto. "La metodología utilizada para el proyecto aplicado me pareció lo más relevante, pues permite generar nuevos proyectos de investigación acción. (...) Es una oportunidad de reflexionar y mejorar las prácticas docentes y directivas" (Participante 119, Curso 2018). 
Figura 4: Valoración de la investigación-acción. La investigación-acción contribuyó al análisis, de un problema relevante de mi institución

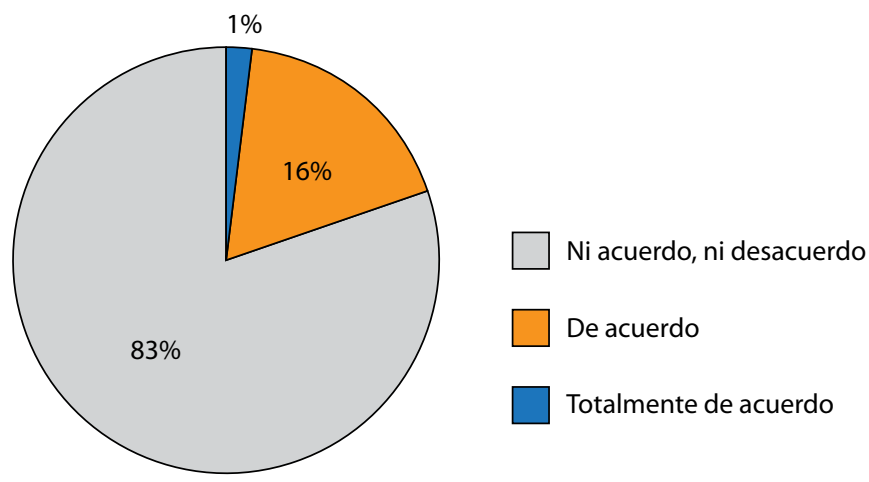

Fuente: Elaboración propia (2019).

Los aspectos de la modalidad $b$-learning que destacan son los siguientes: $100 \%$ valora que los recursos estaban permanentemente disponibles; $87 \%$ considera que el trabajo virtual le proporcionó autonomía y la posibilidad de gestionar el tiempo; $85,4 \%$ señala que las videoconferencias aportaron a la comprensión y claridad de las tareas; $82,1 \%$ señala que el blog fue un buen espacio para compartir y reflexionar sobre el propio trabajo y el de sus compañeros; y en la misma línea, $75,6 \%$ señala que los foros fueron una oportunidad para compartir opiniones e ideas con otros participantes profesores y directivos escolares. "Aprecio en particular el blog como instancia de intercambio con el docente y los compañeros. A su vez, la modalidad a distancia que brinda una solución para la dificultad de acceso a los centros educativos y el poco tiempo que disponemos después del trabajo y el hogar" (Participante 81, Curso 2017). Todos los elementos dan cuenta de cómo la tecnología contribuye al éxito del curso, en la medida que está supeditada a estrategias metodológicas orientadas a la reflexión contextualizada y a la temprana aplicación de los aprendizajes y las habilidades directivas.

Es interesante observar que quienes participaron valoran positivamente el curso en términos generales y se muestran optimistas respecto a su futuro desempeño, 95,1\% considera que la formación recibida es suficiente para que en el futuro pueda transferir los contenidos a su práctica docente/directiva; $95,1 \%$ cree que el curso le ha permitido desarrollar y/o profundizar competencias directivas que no tenía; y $98,4 \%$ considera que los conocimientos y competencias desarrolladas en esta formación mejorará su desempeño profesional.

Figura 5: Valoración de los conocimientos adquiridos para el desempeño laboral.

Utilizar los conocimientos adquiridos o las competencias desarrolladas en esta afirmación, mejorará mi desempeño profesional

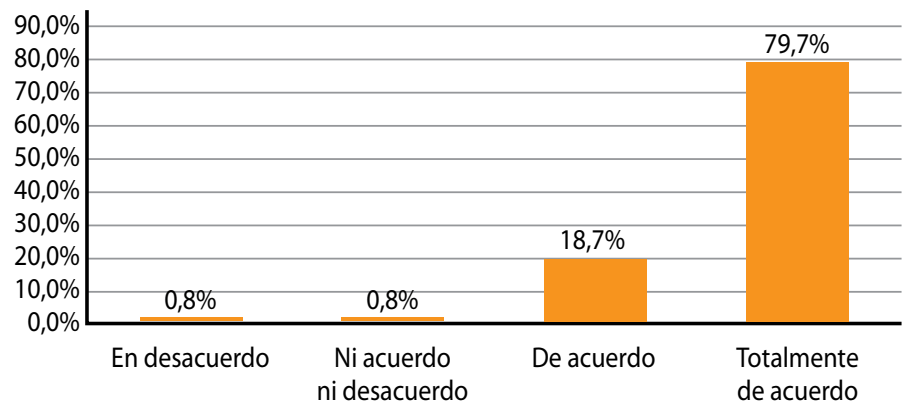

Fuente: Elaboración propia (2019). 


\section{Elementos que favorecen un enfoque aplicado en la formación en liderazgo directivo}

A partir del análisis correlacional emergen algunas relaciones estadísticamente significativas que merecen ser analizadas y que dan cuenta de algunas fortalezas del enfoque aplicado en la formación.

TABLA 1

Análisis correlacional entre dos variables

\begin{tabular}{lcc} 
& & $\begin{array}{c}\text { A través de las actividades y tareas pude } \\
\text { transferir la teoría a situaciones concretas } \\
\text { del ámbito escolar. }\end{array}$ \\
$\begin{array}{l}\text { Las actividades facilitaron la reflexión sobre } \\
\text { la práctica y el contexto educativo }\end{array}$ & $\begin{array}{c}\text { Correlación de Pearson } \\
\text { Sig. (bilateral) }\end{array}$ &, $777^{*}$ \\
& $\mathrm{~N}$ &, 000 \\
\hline
\end{tabular}

* La correlación es significativa al 0,01 (bilateral).

Fuente: Elaboración propia (2019).

Se observó correlación entre la percepción respecto a que las actividades facilitan la reflexión sobre la práctica y el contexto escolar, y que a través de las actividades y tareas del curso se puede transferir la teoría a situaciones concretas del ámbito escolar. Lo que de alguna manera estaría afirmando, desde la percepción de los participantes, que el tipo de actividades propuestas, sustentadas en la reflexión sobre la práctica, favorece la transferencia de los aprendizajes al contexto, tal como se proyectó desde el curso, y como lo evidencia la literatura (Cano, 2015; Grohmann et al., 2014).

La correlación evidenciada entre investigación-acción y reflexión sobre el contexto institucional da cuenta de que se trata de procesos concatenados, percibidos como tales por quienes participaron en el curso, que favorecen la comprensión de su entorno escolar.

TABLA 2

Análisis correlacional entre dos variables

\begin{tabular}{lcc} 
El enfoque aplicado, bajo la investigación- & $\begin{array}{c}\text { Utilizar los conocimientos y competencias } \\
\text { desarrolladas en esta formación mejorarán } \\
\text { mi desempeño profesional }\end{array}$ \\
$\begin{array}{lcc}\text { acción, ayudó al desarrollo de mis compe- } \\
\text { tencias directivas. }\end{array}$ & Sig. (bilateral) &, $820^{*}$ \\
& $\mathrm{~N}$ &, 000 \\
\hline
\end{tabular}

* La correlación es significativa al 0,01 (bilateral)

Fuente: Elaboración propia (2019).

Desde una dimensión proyectiva, la correlación observada entre un enfoque aplicado que ayuda al desarrollo de competencias directivas y la percepción respecto a que la utilización de conocimientos y competencias desarrolladas en el curso mejorarán el desempeño profesional, habla de una propuesta metodológica que genera autoconfianza y que facilita la transferencia de los aprendizajes (Grohmann et al., 2014; Grohmann \& Kauffeld, 2013; Hutchins et al., 2010).

Por último, es importante señalar que no se observó correlación entre las variables analizadas y las características estructurales de quienes participaron en el curso (sexo, años de experiencia y cargo). 


\section{DISCUSIÓN}

A partir de los datos levantados en el estudio, se logró identificar fortalezas en cuatro de los ejes articuladores de la metodología utilizada en el curso. La alta valoración de la aplicabilidad de los aprendizajes es un aspecto que se destaca al ver que se articula con el sentido de una formación en el ejercicio profesional y responde a las críticas permanentes a la formación (Bozu \& Imbernón, 2016; C. Díaz Larenas, Solar Rodríguez, Soto Hernández, \& Conejeros del Solar, 2015; Nieto \& Alfageme-González, 2017).

Así mismo, se demuestra que la metodología formativa sustentada en la investigación-acción, genera aprendizajes, lo que se evidencia en los resultados del pre y post-test. Es interesante constatar la valoración de esta metodología por quienes participaron en el curso, y confirma su relación con el cambio de prácticas que declaran haber desarrollado, lo que se condice con los estudios de Aas (2019), Díaz-Bazo (2017) y Campayo-Muñoz \& Cabedo-Mas (2018). También es atribuible a la investigación-acción y los procesos reflexivos que detona, el desarrollo de su capacidad para toma de decisiones, lo que conversa con el estudio de Van Velzen (2017).

Se valora positivamente el trabajo autónomo y esta modalidad que les otorga flexibilidad para la organización de su tiempo, así como el rol de las videoconferencias y las clases presenciales, los que son atributos de la modalidad $b$-learning (Bocconi \& Trentin, 2014). Modalidad que, cuidando el tipo de actividades que se desarrollan y la adecuada mediación, demuestra efectividad (Dewi, Ciptayani, Surjono, \& Priyanto, 2018; Goode et al., 2018; Martin, Kumar, Abernathy, \& Browne, 2018; Yeop, Yaakob, Wong, Don, \& Zain, 2019).

Su estructuración con base en actividades aplicadas que responden a su enfoque basado en competencias es otro aspecto valorado por las y los participantes del curso y que se condice con lo esperado del enfoque (Cano, 2015; Le Boterf, 2011; Perrenaud, 2001; Romero \& Halal, 2017; Tejada Fernández, 2012; Tejada Fernández \& Ruiz Bueno, 2016; Tobón, 2013). Mientras que la importancia de enfocar las acciones de aprendizaje desde una mirada contextualizada se sabe que garantiza la posibilidad de transferencia al desarrollar autoconfianza en los aprendices (Grohmann et al., 2014; Grohmann \& Kauffeld, 2013; Hutchins et al., 2010), y en este caso es observada en la valoración de los estudiantes, pero también en las correlaciones significativas encontradas.

Por último, la estrategia del aula invertida, propiciada desde la modalidad b-learning, que potenció el trabajo autónomo y la reflexión colaborativa en las clases (Gilboy et al., 2015; Strayer, 2012; Zainuddin \& Halili, 2016) es también considerada un aspecto relevante.

Sin duda, es la articulación de las estrategias lo que le imprime pertinencia a un proceso formativo sustentado en un enfoque aplicado de la formación y que luego facilita la aplicación de los aprendizajes en contextos diversos.

\section{CONCLUSIONES}

La investigación-acción como eje articulador aporta formación de habilidades reflexivas, pero también desencadena procesos de cambio de prácticas, aspecto altamente documentado (Aas et al., 2019; Campayo-Muñoz \& Cabedo-Mas, 2018; Díaz-Bazo, 2017; Van Velzen, 2017) y que se convierte en el sentido final de una formación en ejercicio de la profesión. Este enfoque cautela la contextualización del proceso formativo. Quizás el principal valor del estudio es demostrar su gran aporte dentro de un proceso $b$-learning que cuida el frágil equilibrio de las acciones presenciales y virtuales bajo una perspectiva de aula invertida. 
Si se espera contar con procesos formativos que impacten en el hacer educativo, modificando realmente las prácticas, cada vez es más necesario la integración de los saberes científicos, profesionales y subjetivos en la formación (Cuadra-Martínez \& Castro, 2018), lo que implica reconocer el valor de la acción práctica. Innovar a través de nuevas estrategias formativas que consideren el aprendizaje activo, las acciones vinculadas al contexto, el uso de la tecnología con sentido y la integración de actividades que generen reflexión y metacognición sobre el hacer educativo, es un camino sólidamente fundamentado respecto de sus posibles impactos.

\section{REFERENCIAS}

Aas, M., Vennebo, K.F \& Halvorsen, K.A. (2019). Benchlearning - an action research program for transforming leadership and school practices. Educational Action Research. https://doi.org/10.1080/09 650792.2019.1566084

Amador, J.A. \& Pagés, T. (2014). La formación del profesorado universitario en las universidades españolas y la transferencia de la formación. In E. Cano \& A. Bartolomé (Eds.), Evaluar la formación es posible. (pp. 35-54). Barcelona: Colección Transmedia XXI. Disponible en http://www.Imi.ub.es/ transmedia21/

Azocar, M. \& Cano, E. (2015). Evaluación diferida del Plan de Formación de Directores, a través de la opinión de los participantes insertos en escuelas municipalizadas de la región de Los Ríos-Chile. Universidad de Barcelona.

Barrera, D., Castillo, H. \& Gallardo, A. (2017). Prácticas docentes y su relación con las creencias pedagógicas y el ambiente escolar. In Contextualización de la enseñanza en Chile. Resultados de la encuesta internacional TALIS 2013, OCDE (pp. 113-152). Santiago: Ministerio de Educación Chile. Disponible en https://centroestudios.mineduc.cl/wp-content/uploads/sites/100/2017/07/TALIS_FINAL_ COMPLETO.pdf

Bartolomé, A. (2004). Conceptos Básicos. Blended Learning. Basic Concepts. Pixel Bit. Revista de Medios Y Educación, 23, 7-20. Disponible en https://recyt.fecyt.es/index.php/pixel/article/ view/61237/37251

Bergmann, J. \& Sams, A. (2012). Flip YOUR Classroom Reach Every Student in Every Class Every Day Library of Congress Cataloging-in-Publication Data. Disponible en https://doi.org/10.1111/teth.12165

Bocconi, S. \& Trentin, G. (2014). Modelling blended solutions for higher education: teaching, learning, and assessment in the network and mobile technology era. Educational Research and Evaluation, 20, 516-535. Disponible en https://doi.org/10.1080/13803611.2014.996367

Bozu, Z. \& Imbernón, F. (2016). El presente y el futuro de la formación docente permanente del profesorado universitario The present and future of constant University teachers training. Educación Y Ciencia, 5(45), 94-105. Disponible en http://www.educacionyciencia.org/index.php/ educacionyciencia/article/download/387/pdf_32

Burns, A. \& Westmacott, A. (2018). Teacher to Researcher: Reflections on a New Action Research Program for University EFL Teachers. Profile: Issues in Teachers' Professional Development, 20(1), 15 23. Disponible en https://doi.org/10.15446/profile.v20n1.66236

Campayo, E. \& Cabedo, A. (2018). Investigación-Acción como recurso para la innovación y mejora de la práctica educativa en conservatorios: Una experiencia en las enseñanzas elementales de música en España. Psychology, Society and Education, 10(1), 15-36. Disponible en https://doi.org/10.25115/ psye.v10i1.1767

Cano, E. (2008). La evaluación por competencias en la educación superior. Profesorado. Revista de Currículum Y Formación Del Profesorado, 12(3), 1-16. 
Cano, E. (2015). Evaluación de la formación. Algunas lecciones aprendidas y algunos retos de futuro. Educar, 51(1), 109-125. Disponible en https://doi.org/10.5565/rev/educar.684

Cano, E. (2016). Factores Favorecedores y Obstaculizadores de la Transferencia de la Formación del Profesorado en Educación Superior. REICE. Revista Iberoamericana Sobre Calidad, Eficacia Y Cambio En Educación., 14(2), 133-150. Disponible en https://doi.org/10.15366/reice2016.14.2.008

Carr, W. (1993). Calidad de la enseñanza e Investigación-Acción. Sevilla: Díada Editora.

Cuadra, D., Castro, P. \& Juliá, M. (2018). Tres Saberes en la Formación Profesional por Competencias : Integración de Teorías Subjetivas, Profesionales y Científicas. Formación Universitaria, 11(5), 19-30.

Cui, G. \& Hu, J. (2018). Experimental Study to Develop Writing skills through Blended Learning in the Times of Internet +, MATEC Web of Conferences 246, 03031. Disponible en https://doi.org/10.1051/ matecconf/201824603031

Dewi, K. C., Ciptayani, P. I., Surjono, H. D., \& Priyanto. (2018). Critical Success Factor for Implementing Vocational Blended Learning. Journal of Physics: Conference Series, 953(1). Disponible en https:// doi.org/10.1088/1742-6596/953/1/012086

Díaz, C. (2017). La investigación-acción en la educación básica en Iberoamérica. Una revisión de la literatura. Magis, Revista Internacional de Investigación En Educación, 10(20), 159-182. https://doi. org/10.11144/Javeriana.m10-20.iaeb

Díaz de Rada, V. \& Domínguez, J. A. (2017). Comparación de métodos de campo en la encuesta / Comparison of Survey Data Collection Methods. Revista Española de Investigaciones Sociológicas, 137-148. Disponible en https://doi.org/10.5477/cis/reis.158.137

Díaz, C., Solar, M. I., \& Soto, V. (2015). Temas clave en la formación de profesores en Chile desde la perspectiva de docentes y directivos. Revista Complutense de Educación Núm, 543(3), 1130-2496. Disponible en https://doi.org/10.5209/rev_RCED.2015.v26.n3.44300

Díaz, C., Solar, M. I., Soto, V. \& Conejeros del Solar, M. (2015). Formación docente en Chile: Perceipción de profesores del sistema escolar y docentes universitarios. Civilizar, 15(28), 229-246. Disponible en http://www.scielo.org.co/pdf/ccso/v15n28/v15n28a16.pdf

Faturrahman, M., Ibrahim, Kahar, M. S., Arsyad, R. \& Rawi, R. D. P. (2018). Development of Learning Media Based on MOODLE Integrated with Blended Learning in Mathematic Learning Process at SMA Muhammadiyah AI-Amin Sorong. IOP Conference Series: Earth and Environmental Science, 175(1). Disponible en https://doi.org/10.1088/1755-1315/175/1/012202

Feixas, M., Marquez, D. \& Sabaté, S. (2014). Experiencia de evaluación de la transferencia de la formación del profesorado universitario: la aplicación del Cuestionario de Transferencia de la Formación Docente en la Universitat Autónoma de Barcelona. In E. Cano \& A. Bartolomé (Eds.), Evaluar la formación es posible. (pp. 53-82). Barcelona: Colección Transmedia XXI.

Garrison, R. (2011). E-Learning in the 21st Century A Framework for Research and Practice. New York: Routledge.

Gegenfurtner, A. (2011). Motivation and transfer in professional training: A meta-analysis of the moderating effects of knowledge type, instruction, and assessment conditions. Educational Research Review, 6(3), 153-168. Disponible en https://doi.org/10.1016/j.edurev.2011.04.001

Gilboy, M. B., Heinerichs, S. \& Pazzaglia, G. (2015). Enhancing student engagement using the flipped classroom. Journal of Nutrition Education and Behavior, 47(1), 109-114. Disponible en https://doi. org/10.1016/j.jneb.2014.08.008

Goode, C., Lamoreaux, M., Atchison, C., Jeffress, E., Lynch, H. \& Sheehan, E. (2018). Quantitative Skills, Critical Thinking, and Writing Mechanics in Blended Versus Face-to-Face Versions of a Research Methods and Statistics Course. Teaching of Psychology, 45(2). Disponible en https://doi.org/https:// doi.org/10.1177/0098628318762873 
Grohmann, A., Beller, J. \& Kauffeld, S. (2014). Exploring the critical role of motivation to transfer in the training transfer process. International Journal of Training and Development, 18(2), 84-103. Disponible en https://doi.org/10.1111/ijtd.12030

Grohmann, A. \& Kauffeld, S. (2013). Evaluating training programs: Development and correlates of the Questionnaire for Professional Training Evaluation. International Journal of Training and Development, 17(2), 135-155. Disponible en https://doi.org/10.1111/ijtd.12005

Hattie, J. (2009). Visible Learning: A Synthesis of Over 800 Meta-analyses Relating to Achievement. New York: Routledge.

Herreid, C. \& Schiller, N. (2013). Cases_Flipped_Classroom. Journal of College Science Teaching, 42(5), 62-66.

Huberman, M. (1990). Las fases de la profesión docente. Ensayo de descripción y previsión. Qurriculum: Revista de teoría, investigación y práctica educativa, 2, 1-9. Disponible en http://www.quadernsdigitals.net/index.php?accionMenu=hemeroteca.VisualizaArticulolU.visualiza\&articulo_id=2638

Hutchins, H.M., Burke, L.A. \& Berthelsen, A.M. (2010). Inside the Black Box: Raising Standards Through Classroom Assessment. Human Resource Management, 49(4), 599-618. Disponible en https://doi. org $/ 10.1002 / \mathrm{hrm}$

Kemmis, S. \& McTaggart, R. (1988). Cómo planificar la Investigación-Acción. Barcelona: Laertes.

Le Boterf, G. (2011). Repenser la Competence. Paris: Eyrolles.

Lee, K., \& Lai, Y. (2017). Facilitating higher-order thinking with the flipped classroom model: a student teacher's experience in a Hong Kong secondary school. Research and Practice in Technology Enhanced Learning, 12(1), 8. Disponible en https://doi.org/10.1186/s41039-017-0048-6.

Lepe, C., Castillo, P., Cerda, G., Montaña, V. \& Recabarren, C. (2014). Experiencia de evaluación de la transferencia de la formación docente: descripción del instrumento de evaluación y resultados hallados en la Universidad Católica del Norte. Antofagasta-Chile. In E. Cano \& A. Bartolomé (Eds.), Evaluar la formación es posible. (pp. 103-118). Barcelona: Colección Transmedia XXI.

Ley No20.903. Diario Oficial de la República de Chile, Santiago, Chile, 4 de marzo 2016. Disponible en https://www.leychile.cl/Navegar?idNorma=1087343.

Limniou, M., Schermbrucker, I., \& Lyons, M. (2018). Traditional and flipped classroom approaches delivered by two different teachers: the student perspective. Education and Information Technologies, 23(2), 797-817. Disponible en https://doi.org/10.1007/s10639-017-9636-8

Martin, P., Kumar, S., Abernathy, L. J. \& Browne, M. (2018). Good, bad or indifferent: A longitudinal multimethods study comparing four modes of training for healthcare professionals in one Australian state. BMJ Open, 8(8), 1-8. Disponible en https://doi.org/10.1136/bmjopen-2017-021264

Nieto, J. M. \& Alfageme-González, M. B. (2017). Enfoques, metodologías y actividades de formación docente. Profesorado. Revista de Currículum Y Formación Del Profesorado, 21(3). Disponible en https://recyt.fecyt.es/index.php/profesorado/article/view/59789

Perrenaud, P. (2004). Diez nuevas competencias para enseñar. Barcelona: Grao.

Romero, R. \& Halal, C. (2017). Aportes del E-Portafolio para la evaluación de competencias. In Experiencias en la implementación de la gestión del talento humano desde el pensamiento complejo (pp. 216-231). Florida: Kresearch Group.

Rowell, L., Polush, E., Riel, E. \& Bruewer, A. (2015). Action researchers' perspectives about the distinguishing characteristics of action research: a Delphi and learning circles mixed-methods study. Educational Action Research, 23(2), 243-270. Disponible en https://doi.org/10.1080/09650792.2014 .990987 
Simon, J., Benedí, C., Blanché, C. \& Bosch, M. (2016). La semipresencialidad en Educación Superior: casos de estudio en los grados de la universidad de Barcelona. Edutec. Revista Electrónica de Tecnología Educativa, 58, 15-33.

Strayer, J. F. (2012). How learning in an inverted classroom influences cooperation, innovation and task orientation. Learning Environments Research, 15(2), 171-193. Disponible en https://doi.org/10.1007/ s10984-012-9108-4

Tejada, J. (2012). La alternancia de contextos para la adquisición de competencias profesionales en escenarios complementarios de educación superior: Marco y estrategia. Educacion XX1, 15(2), 17-40. Disponible en https://doi.org/10.5944/educxx1.15.2.125

Tejada, J. \& Ruiz, C. (2016). Evaluación de competencias profesionales en educación superior: Retos e implicaciones. Educacion XX1, 19(1), 17-38. Disponible en https://doi.org/10.5944/educXX1.12175

Tobón, S. (2013). Formación integral y competencias: pensamiento complejo, curriculo, didáctica y evaluación. Bogotá: Ecoe.

Van Velzen, J. (2017). Metacognitive Knowledge:Development, Application, and Improvement, Information. USA: Age Publ. Inc.

Yeop, M., Yaakob, M., Wong, K., Don, Y. \& Zain, F. (2019). Implementation of ICT policy (Blended Learning Approach): Investigating factors of behavioural intention and use behaviour. International Journal of Instruction, 12(1), 767-782. Disponible en https://doi.org/10.29333/iji.2019.12149a

Zainuddin, Z. \& Halili, S. (2016). Flipped classroom research and trends from different fields of study. He International Review of Research in Open and Distributed Learning, 17(3). Disponible en https://doi. org/http://dx.doi.org/10.19173/irrodl.v17i3.2274.

\section{Para citar este documento:}

Romero, R., Montt, B., Maregatti, C., Gracia, M. y Segura, D. (2020). Investigación-acción, aula invertida y formación basada en competencias. Elementos clave para una efectiva formación b-learning en liderazgo directivo. Revista Innovaciones Educativas. 22(32), 21-35. DOI: 10.22458/ie.v22i32.2896 